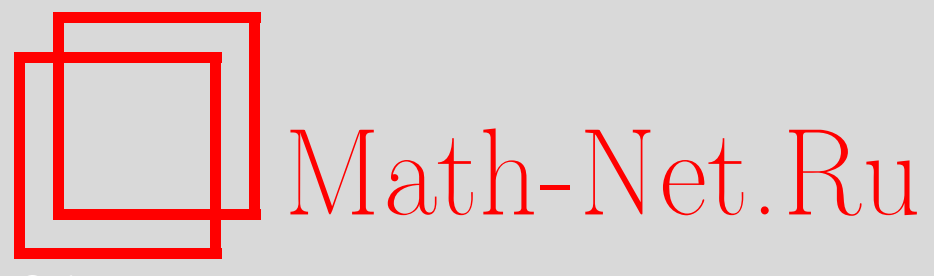

Х. А. Хачатрян, K вопросу разрешимости одного класса интегро-дифференциальных уравнений первого порядка с переменными коэффициентами, Матем. заметки, 2008, том 83, выпуск 6, 933-940

DOI: https://doi.org/10.4213/mzm4835

Использование Общероссийского математического портала Math-Net.Ru подразумевает, что вы прочитали и согласны с пользовательским соглашением http: //www . mathnet.ru/rus/agreement

Параметры загрузки:

IP: 3.85 .73 .92

26 апреля 2023 г., 18:31:58

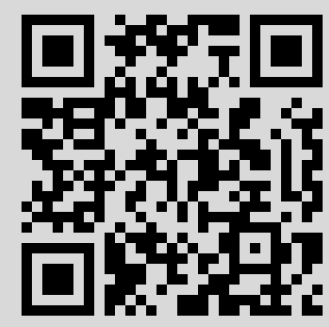


Том 83 выпуск 6 июнь 2008

УДК 517.9

\section{K вопросу разрешимости одного класса интегро-дифференциальных уравнений первого порядка с переменными коэффициентами}

\section{Х. А. Хачатрян}

В настоящей заметке рассматривается один класс линейных интегро-дифференциальных уравнений первого порядка со стохастическим ядром и с переменными коэффициентами на полуоси. Рассмотренные уравнения имеют важные применения в физической кинетике. Сочетание специальных факторизационных методов с методами интегральных уравнений Фредгольма второго рода дают возможность построить решение этих уравнений в пространстве Соболева $W_{1}^{1}\left(\mathbb{R}^{+}\right)$. В некоторых особых случаях удается описать также структуру построенных решений.

Библиография: 7 названий.

1. Введение. Рассмотрим задачу типа Коши для следующего интегро-дифференциального уравнения на полуоси:

$$
\begin{gathered}
\frac{d f}{d x}+\lambda(x) \int_{0}^{\infty} K(x, t) f(t) d t+g(x)=q(x) f(x), \quad x>0, \\
f(0)=\rho_{0} \in(0,+\infty)
\end{gathered}
$$

где $\lambda, q \in L_{\infty}(0,+\infty)$, ядро $K(x, t)$ - неотрицательная функция, удовлетворяющая условию стохастичности:

$$
\sup _{t \in(0,+\infty)} \int_{0}^{\infty} K(x, t) d x=1,
$$

а свободный член уравнения (1) удовлетворяет условию

$$
0 \leqslant g \in L_{1}(0,+\infty) .
$$

Изучение задачи (1)-(4) представляет не только самостоятельный математический интерес, но и играет важную роль в вопросах решения различных актуальных задач физической кинетики. Из них наиболее известной является задача распределения электрического поля в полубесконечном металле [1], [2].

Первые результаты по изучению уравнения (1), в том частном случае, когда

$$
q(x) \equiv 0, \quad \lambda(x)=c \equiv \text { const }, \quad K(x, t)=K(x-t),
$$


где

$$
K(x)=\int_{1}^{\infty} e^{-|x| s} \frac{d s}{s^{2}}
$$

появились в работах [2], [3].

Далее, когда

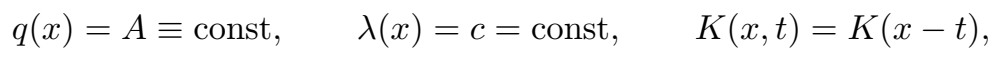

$0 \leqslant K \in L_{1}(-\infty,+\infty), \|\left. K\right|_{L_{1}}=1$, уравнение рассматривалось в работе [4], где при наложении некоторых дополнительных условий на параметры $A$ и $c$ была доказана его разрешимость в $W_{1}^{1}(0,+\infty)$ (где $W_{p}^{n}(0,+\infty)$ состоит из функций $f$ таких, что $\left.f^{(k)} \in L_{p}(0,+\infty), k=0,1,2, \ldots, n\right)$. В некоторых частных случаях была найдена структура построенных решений.

В настоящей работе получены следующие результаты.

ТЕОРема. Предположим, что ядро $K(x, t)$ и свободный член уравнения (1), удовлетворяют соответственно условиям (3) $u(4), a 0 \leqslant \lambda, q \in L_{\infty}(0,+\infty)$. Тогда, если существует число $\alpha>0$ такое, что

$$
\sup _{y \in(0, \infty)} \int_{0}^{\infty} \lambda(x) K(x, y) d x+\sup _{y \in(0, \infty)}(\alpha-q(y))\left(1-e^{-\alpha y}\right)<\alpha,
$$

то задача (1)-(4) имеет положительное, абсолютно непрерывное решение; более mого, $f \in W_{1}^{1}(0,+\infty)$.

ЗАмЕчАниЕ 1. Нетрудно убедиться, что если, например,

$$
\sup _{x \in(0, \infty)} \lambda(x)<\inf _{x \in(0, \infty)} q(x),
$$

то условие (6) теоремы автоматически выполняется.

ПРЕДЛОЖЕНИЕ. Пусть ядро $K(x, t)$ и свободный член $g(x)$ уравнения (1), удовлетворяют соответственно условиям (3) u (4), a $\lambda, q \in L_{\infty}(0,+\infty)$. Тогда, если существует число $\beta>0$ такое, что существует ограниченный обратный оператор для $I+W_{\beta}$ в $L_{1}(0,+\infty)$, где $W_{\beta}$ - интегралъный оператор вида

$$
\left(W_{\beta} f\right)(x)=\int_{0}^{\infty} W_{\beta}(x, t) f(t) d t, \quad f \in L_{1}(0,+\infty),
$$

с ядром

$$
W_{\beta}(x, y)=\lambda(x) \int_{0}^{\infty} e^{-\beta \tau} K(x, \tau+y) d \tau-(\beta+q(x)) e^{-\beta(x-y)} \theta(x-y),
$$

где

$$
\theta(x)= \begin{cases}1, & \text { если } x \geqslant 0 \\ 0, & \text { если } x<0\end{cases}
$$

то задача (1)-(4) в пространстве $W_{1}^{1}(0,+\infty)$ обладает решением следующей струкmуры:

$$
f(x)=\rho_{0} e^{-\beta x}+\int_{0}^{x} e^{-\beta(x-t)} F(t) d t, \quad \text { əde } \quad F \in L_{1}(0,+\infty) .
$$


2. Обозначения и вспомогательные леммы. Перепишем задачу (1), (2) в операторной форме:

$$
\left\{\begin{array}{l}
(D-Q+\Lambda \mathscr{K}) f+g=0, \\
f(0)=\rho_{0}>0
\end{array}\right.
$$

где $(D f)(x)=d f / d x$ - оператор дифференцирования, $(Q f)(x)=q(x) f(x),(\Lambda f)(x)=$ $\lambda(x) f(x)$ - операторы умножения, а

$$
(\mathscr{K} f)(x)=\int_{0}^{\infty} K(x, t) f(t) d t, \quad x>0 .
$$

Пусть $E^{+}$- одно из следующих банаховых пространств: $L_{p}(0,+\infty), 1 \leqslant p \leqslant+\infty$. Предполагается, что оператор $\mathscr{K}$ непрерывно действует в $E^{+}$.

Обозначим через $\Omega$ класс следующих интегральных операторов: $\mathscr{K} \in \Omega$, если

$$
\begin{aligned}
& \mu_{1}=\sup _{t \in(0, \infty)} \int_{0}^{\infty}|K(x, t)| d x<+\infty, \\
& \mu_{2}=\sup _{x \in(0, \infty)} \int_{0}^{\infty}|K(x, t)| d t<+\infty .
\end{aligned}
$$

Легко проверить, что класс $\Omega$ является алгеброй. Действительно, пусть, например, $\mathscr{K}, H \in \Omega, W \equiv \mathscr{K} H$. Тогда

$$
\begin{aligned}
\int_{0}^{\infty}|W(x, y)| d y= & \int_{0}^{\infty}\left|\int_{0}^{\infty} K(x, t) h(t, y) d t\right| d y \leqslant \int_{0}^{\infty} \int_{0}^{\infty}|K(x, t)||h(t, y)| d t d y \\
= & \int_{0}^{\infty}|K(x, t)| \int_{0}^{\infty}|h(t, y)| d y d t \leqslant \sup _{t \in(0, \infty)} \int_{0}^{\infty}|h(t, y)| d y \\
& \times \sup _{x \in(0, \infty)} \int_{0}^{\infty}|K(x, t)| d t<+\infty
\end{aligned}
$$

откуда следует, что

$$
\sup _{x \in(0, \infty)} \int_{0}^{\infty}|W(x, y)| d y<+\infty .
$$

Точно так же доказывается, что

$$
\sup _{y \in(0, \infty)} \int_{0}^{\infty}|W(x, y)| d x<+\infty .
$$

Из (13), (14) следует, что

$$
\|\mathscr{K}\|_{L_{1}} \leqslant \mu_{1}, \quad\|\mathscr{K}\|_{L_{\infty}} \leqslant \mu_{2} .
$$

Согласно интерполяционной теореме Рисса [5] имеем

$$
\|\mathscr{K}\|_{L_{p}} \leqslant \max \left(\mu_{1}, \mu_{2}\right), \quad 1<p<+\infty .
$$

Рассмотрим следующую задачу факторизации: для операторов $D, Q, \Lambda$ и $\mathscr{K}$, и для каждого $\alpha>0$ найти такой интегральный оператор $B_{\alpha}$, действующий в $W_{1}^{1}(0,+\infty)$, чтобы

$$
D-Q+\Lambda \mathscr{K}=(D-\alpha I)\left(I-B_{\alpha}\right),
$$

где $I$ - единичный оператор. Факторизацию (17) мы будем понимать как равенство операторов, действующих в $W_{1}^{1}(0,+\infty)$. 


\section{Имеет место}

Лемма 1. Пусть $\mathscr{K}$ - интегралъный оператор (3), (12). Тогда для каждого $\alpha>0$ справедливо (17) как равенство операторов, действующих в $W_{1}^{1}(0,+\infty)$. Ядерная бункиия $b_{\alpha}(x, y)$ оператора $B_{\alpha}$ имеет вид

$$
b_{\alpha}(x, y)=\int_{0}^{\infty} e^{-\alpha \tau} K(x+\tau, y) \lambda(x+\tau) d \tau+(\alpha-q(y)) e^{-\alpha(y-x)} \theta(y-x) .
$$

Далее, если $\mathscr{K} \in \Omega$, то $B_{\alpha} \in \Omega$ длякаждого $\alpha>0$.

ДоказАТЕЛьство. Имеем

$$
D-Q+\Lambda \mathscr{K}=D-\alpha I+\alpha I-Q+\Lambda \mathscr{K}=(D-\alpha I)\left(I-B_{\alpha}\right),
$$

где

$$
B_{\alpha}=\Gamma_{\alpha} \Lambda \mathscr{K}-\Gamma_{\alpha} Q+\alpha \Gamma_{\alpha},
$$

а $\Gamma_{\alpha}$ - обратный оператор для оператора $\alpha I-D$ в пространстве $W_{1}^{1}(0,+\infty)$, причем

$$
\left(\Gamma_{\alpha} f\right)(x)=\int_{x}^{\infty} e^{-\alpha(t-x)} f(t) d t
$$

Путем перехода в формуле (20) от операторных равенств к ядрам с учетом (21) получим, что ядро оператора $B_{\alpha}$ имеет вид (18). Так как $\lambda \in L_{\infty}(0,+\infty)$ и

$$
b_{\alpha}(x, y)=\int_{x}^{\infty} e^{-\alpha(z-x)} K(z, y) \lambda(z) d z+(\alpha-q(y)) e^{-\alpha(y-x)} \theta(y-x),
$$

то нетрудно убедиться, что оператор $B_{\alpha}$ действует в $W_{1}^{1}(0,+\infty)$. Перейдем ко второй части доказательства леммы. Пусть $\mathscr{K} \in \Omega$. Докажем, что $B_{\alpha} \in \Omega$. Имеем

$$
\begin{aligned}
\int_{0}^{\infty}\left|b_{\alpha}(x, y)\right| d y \leqslant & \int_{0}^{\infty}\left|\int_{0}^{\infty} e^{-\alpha \tau} \lambda(x+\tau) K(x+\tau, y) d \tau\right| d y+\int_{x}^{\infty}|\alpha-q(y)| e^{-\alpha(y-x)} d y \\
\leqslant & \int_{0}^{\infty} e^{-\alpha \tau} \int_{0}^{\infty}|\lambda(x+\tau)||K(x+\tau, y)| d y d \tau+\frac{1}{\alpha} \sup _{y \in(0,+\infty)}|\alpha-q(y)| \\
& \leqslant \sup _{x \in(0,+\infty)}|\lambda(x)| \int_{0}^{\infty} e^{-\alpha \tau} \int_{0}^{\infty}|K(x+\tau, y)| d y d \tau \\
& +\frac{1}{\alpha} \sup _{y \in(0, \infty)}|\alpha-q(y)| \\
& \leqslant \frac{\mu_{2}}{\alpha} \sup _{x \in(0,+\infty)}|\lambda(x)|+\frac{1}{\alpha} \sup _{y \in(0, \infty)}|\alpha-q(y)|<+\infty,
\end{aligned}
$$

откуда следует, что

$$
\sup _{x \in(0,+\infty)} \int_{0}^{\infty}\left|b_{\alpha}(x, y)\right| d y<+\infty .
$$

Аналогичными рассуждениями проверяется, что

$$
\sup _{y \in(0, \infty)} \int_{0}^{\infty}\left|b_{\alpha}(x, y)\right| d x<+\infty .
$$

Лемма доказана. 
Точно так же доказывается следующая

Лемма 2. Предположим, что $\mathscr{K}$ - интегральный оператор вида (3), (12). Тогда для каждого $\beta>0$ имеет место факторизация

$$
D-Q+\Lambda \mathscr{K}=\left(I+W_{\beta}\right)(D+\beta I)
$$

как равенство операторов, действующих в $W_{1}^{1}(0,+\infty)$, где $W_{\beta}-$ интегральный оператор вида (8), (9). Далее, если $\mathscr{K} \in \Omega$, то $W_{\beta} \in \Omega$ для каждого $\beta>0$.

ЗАмЕчАниЕ 2. В дальнейшем, если не будет оговорено противное, мы будем считать, что

$$
\alpha \geqslant \sup _{x \in(0,+\infty)} q(x)
$$

Тогда $b_{\alpha}(x, y) \geqslant 0,(x, y) \in \mathbb{R}^{+} \times \mathbb{R}^{+}$.

3. Уменьшение спектрального радиуса оператора $B_{\alpha}$. Обозначим через $r(A)$ спектральный радиус оператора $A \in \Omega$. Пусть $B_{\alpha}^{+}$и $B_{\alpha}^{-}-$вольтерровы части оператора $B_{\alpha}$ :

$$
\left(B_{\alpha}^{+} f\right)(x)=\int_{0}^{x} b_{\alpha}(x, t) f(t) d t, \quad\left(B_{\alpha}^{-} f\right)(x)=\int_{x}^{\infty} b_{\alpha}(x, t) f(t) d t
$$

Имеет место следующая

Теорема (об уменьшении $r\left(B_{\alpha}\right)$ ). Пусть $\mathscr{K} \in \Omega$ и операторы $I-B_{\alpha}^{ \pm}$обратимъ при некотором значении $\alpha>0$. Тогда имеет место факторизация

$$
D-Q+\Lambda \mathscr{K}=(D-\alpha I)\left(I-B_{\alpha}^{-}\right)\left(I-G_{\alpha}\right)\left(I-B_{\alpha}^{+}\right)
$$

где $G_{\alpha}=\Phi_{\alpha}^{+} \Phi_{\alpha}^{-} \in \Omega,\left(I-B_{\alpha}^{ \pm}\right)^{-1}=I+\Phi_{\alpha}^{ \pm}$. Далее, если $r\left(B_{\alpha}\right)<1, \operatorname{mor}\left(G_{\alpha}\right)<r\left(B_{\alpha}\right)<1$.

ДокАЗАТЕЛьство. Представим оператор $I-B_{\alpha}$ в виде

$$
I-B_{\alpha}=I-B_{\alpha}^{-}-B_{\alpha}^{+}+B_{\alpha}^{-} B_{\alpha}^{+}-B_{\alpha}^{-} B_{\alpha}^{+}=\left(I-B_{\alpha}^{-}\right)\left(I-B_{\alpha}^{+}\right)-B_{\alpha}^{-} B_{\alpha}^{+} .
$$

Нетрудно убедиться, что

$$
\left(I-B_{\alpha}^{-}\right)^{-1} B_{\alpha}^{-}=\Phi_{\alpha}^{-}, \quad B_{\alpha}^{+}\left(I-B_{\alpha}^{+}\right)^{-1}=\Phi_{\alpha}^{+} .
$$

Таким образом, из (17) с учетом (26) и (27) приходим к факторизации (25). Теперь докажем, что если $r\left(B_{\alpha}\right)<1$, то $r\left(G_{\alpha}\right)<r\left(B_{\alpha}\right)<1$. Пусть $\xi$ - неподвижная точка оператора $G_{\alpha}: G_{\alpha} \xi=\nu \xi$, где $\|\xi\|_{E(0, \infty)}=1$ (очевидно, что последнего всегда можно достичь). Обозначим через $\eta$ решение уравнения $\left(I-B_{\alpha}^{+}\right) \eta=\xi$, тогда $\eta^{*}$ (сопряженное к $\eta$ ) будет удовлетворять уравнению $\eta^{*}\left(I-B_{\alpha}^{-}\right)=\xi^{*}$, причем $\|\eta\|_{E(0, \infty)}>1=\|\xi\|_{E(0, \infty)}$. Тогда из разложения

$$
I-B_{\alpha}=\left(I-B_{\alpha}^{-}\right)\left(I-G_{\alpha}\right)\left(I-B_{\alpha}^{+}\right)
$$

с учетом $G_{\alpha} \xi=\nu \xi$ будем иметь

$$
\|\eta\|_{E(0, \infty)}^{2}-\eta^{*} B_{\alpha} \eta=1-\nu .
$$


Но с другой стороны, используя известный вариационный принцип [6], получим

$$
\eta^{*} B_{\alpha} \eta \leqslant \eta\left(B_{\alpha}\right)\|\eta\|_{E(0, \infty)}^{2} .
$$

Следовательно, из (28) с учетом (29) будем иметь

$$
1-\nu \geqslant\|\eta\|_{E(0, \infty)}^{2}\left(1-r\left(B_{\alpha}\right)\right), \quad\|\eta\|_{E(0, \infty)}^{2}>1 .
$$

Итак, из последнего неравенства получим $r\left(G_{\alpha}\right)<r\left(B_{\alpha}\right)<1$. Теорема доказана.

\section{4. Доказательство основных результатов.}

ДоКАЗАТЕЛЬСТво тЕОРЕмЫ. Используя факторизацию (17), уравнение (1) перепишем в следующей форме:

$$
(D-\alpha I)\left(I-B_{\alpha}\right) f=-g .
$$

Решение этого уравнения сводится к последовательному решению следующих двух связанных уравнений:

$$
\begin{gathered}
(D-\alpha I) \psi=-g, \\
\left(I-B_{\alpha}\right) f=\psi
\end{gathered}
$$

Поскольку $0 \leqslant g \in L_{1}(0,+\infty)$, уравнение $(31)$ в $W_{1}^{1}(0,+\infty)$ имеет положительное решение вида

$$
0 \leqslant \psi(x)=\int_{x}^{\infty} e^{-\alpha(t-x)} g(t) d t \in W_{1}^{1}(0, \infty) .
$$

Уравнение (32) перепишем в раскрытой форме:

$$
f(x)=\psi(x)+\int_{0}^{\infty} b_{\alpha}(x, y) f(y) d y
$$

и применим к нему следующий итерационный процесс:

$$
f^{(n+1)}(x)=\psi(x)+\int_{0}^{\infty} b_{\alpha}(x, y) f^{(n)}(y) d y, \quad n=0,1,2, \ldots, \quad f^{(0)}=\psi(x) .
$$

Поскольку $0 \leqslant \psi \in W_{1}^{1}(0,+\infty), b_{\alpha}(x, y) \geqslant 0$, из (34) с учетом (18) следует, что

$$
\text { 1) } \psi(x) \leqslant f^{(n)} \uparrow \quad \text { по } n ; \quad \text { 2) } f^{(n)} \in W_{1}^{1}(0,+\infty) .
$$

Оценим следующий интеграл:

$\int_{0}^{\infty} b_{\alpha}(x, y) d x \leqslant \int_{0}^{\infty} d x \int_{0}^{\infty} e^{-\alpha \tau} \lambda(x+\tau) K(x+\tau, y) d \tau+\frac{1}{\alpha} \sup _{y \in(0,+\infty)}(\alpha-q(y))\left(1-e^{-\alpha y}\right)$.

Изменяя порядок интегрирования в последнем повторном интеграле, получим

$$
\begin{aligned}
\int_{0}^{\infty} b_{\alpha}(x, y) d x & \leqslant \int_{0}^{\infty} e^{-\alpha \tau} \int_{0}^{\infty} \lambda(z) K(z, y) d z d \tau+\frac{1}{\alpha} \sup _{y \in(0,+\infty)}(\alpha-q(y))\left(1-e^{-\alpha y}\right) \\
& \leqslant \frac{1}{\alpha}\left(\sup _{y \in(0,+\infty)} \int_{0}^{\infty} \lambda(z) K(z, y) d z+\sup _{y \in(0,+\infty)}(\alpha-q(y))\left(1-e^{-\alpha y}\right)\right) .
\end{aligned}
$$


Следовательно, из последнего неравенства с учетом (6) будем иметь

$$
\sup _{y \in(0,+\infty)} \int_{0}^{\infty} b_{\alpha}(x, y) d x \leqslant \frac{\rho(\alpha)}{\alpha}<1
$$

где

$$
\rho(\alpha)=\sup _{y \in(0,+\infty)} \int_{0}^{\infty} \lambda(z) K(z, y) d z+\sup _{y \in(0,+\infty)}(\alpha-q(y))\left(1-e^{-\alpha y}\right) .
$$

Из оценки (35) с учетом (34) получим

$$
\begin{aligned}
\int_{0}^{\infty} f^{(n+1)}(x) d x & \leqslant \int_{0}^{\infty} \psi(x) d x+\frac{\rho(\alpha)}{\alpha} \int_{0}^{\infty} f^{(n)}(y) d y \\
& \leqslant \int_{0}^{\infty} \psi(x) d x+\frac{\rho(\alpha)}{\alpha} \int_{0}^{\infty} f^{(n+1)}(y) d y
\end{aligned}
$$

откуда

$$
\int_{0}^{\infty} f^{(n+1)}(x) d x \leqslant \frac{\alpha \int_{0}^{\infty} \psi(x) d x}{\alpha-\rho(\alpha)}=\frac{\int_{0}^{\infty}\left(1-e^{-\alpha t}\right) g(t) d t}{\alpha-\rho(\alpha)} .
$$

Следовательно, из теоремы Леви [7] заключаем, что последовательность $\left\{f^{(n)}(\tau)\right\}_{1}^{\infty}$ сходится почти всюду в $(0, \infty)$ к суммируемой функции $f$, этот предел и есть решение уравнения (33), причем

$$
\frac{1}{\alpha} \int_{0}^{\infty}\left(1-e^{-\alpha t}\right) g(t) d t \leqslant \int_{0}^{\infty} f(y) d y \leqslant \frac{1}{\alpha-\rho(\alpha)} \int_{0}^{\infty}\left(1-e^{-\alpha t}\right) g(t) d t .
$$

Поскольку $\lambda \in L_{\infty}(0, \infty), b(\cdot, y) \in W_{1}^{1}(0,+\infty)$ по $x$ при каждом фиксированном $y \in(0, \infty)$, из $(33)$ видно, что $f \in W_{1}^{1}(0,+\infty)$. Теорема доказана.

ЗАмЕчАНИЕ 3. Для улучшения скорости сходимости итерации (34) целесообразно использовать факторизацию $(25)$, так как $r\left(G_{\alpha}\right)<r\left(B_{\alpha}\right)<1$. Тогда решение уравнения (1) сводится к последовательному решению следующих четырех связанных уравнений:

$$
(D-\alpha I) \psi=-g, \quad\left(I-B_{\alpha}^{-}\right) \varphi=\psi, \quad\left(I-G_{\alpha}\right) \chi=\varphi, \quad\left(I-B_{\alpha}^{+}\right) f=\chi .
$$

ДоКАЗАТЕЛЬСтво ПРЕДЛОЖЕНИЯ. Факторизация (22) сводит уравнение (1) к решению следующих двух связанных уравнений:

$$
\begin{gathered}
\left(I+W_{\beta}\right) F=-g, \\
(D+\beta I) f=F .
\end{gathered}
$$

Поскольку оператор $\left(I+W_{\beta}\right)$ имеет ограниченный обратный в $L_{1}(0, \infty)$ при некотором значении $\beta>0$ и $g \in L_{1}(0, \infty)$, то $F \in L_{1}(0, \infty)$. Перепишем уравнение (39) в раскрытой форме и присоеденим к нему начальное значение $(2)$ :

$$
\left\{\begin{array}{l}
f^{\prime}(x)+\beta f(x)=F(x), \\
f(0)=\rho_{0} \in(0, \infty)
\end{array}\right.
$$

Легко можно убедиться, что задача Коши (40) имеет единственное решение вида

$$
f(x)=\int_{0}^{x} e^{-\beta(x-t)} F(t) d t+\rho_{0} e^{-\beta x} .
$$


Нетрудно убедиться также, что $f \in W_{1}^{1}(0, \infty)$. Действительно, поскольку $F \in$ $L_{1}(0,+\infty)$, в силу теоремы Фубини [7] для каждого $r>0$ будем иметь

$$
\int_{0}^{r}|f(x)| d x \leqslant \int_{0}^{r}|F(t)| \int_{t}^{r} e^{-\beta(x-t)} d x d t+\frac{\rho_{0}}{\beta}\left(1-e^{-\beta r}\right) \leqslant \frac{1}{\beta} \int_{0}^{\infty} \mid F(t) d t+\frac{\rho_{0}}{\beta},
$$

откуда

$$
\int_{0}^{\infty}|f(x)| d x<+\infty
$$

Абсолютно аналогичными рассуждениями проверяется, что

$$
f^{\prime}(x)=F(x)-\beta \int_{0}^{x} e^{-\beta(x-t)} F(t) d t-\rho_{0} \beta e^{-\beta x} \in L_{1}(0, \infty) .
$$

Предложение доказано.

Выражаю искреннюю признательность профессору Н. Б. Енгибаряну и В. Маргаряну за полезные обсуждения.

\section{СПИСОК ЦИТИРОВАННОЙ ЛИТЕРАТУРЫ}

[1] Е. М. Лифшиц, Л. М. Питаевский, Физическая кинетика, Теоретическая физика, 10, Наука, М., 1979.

[2] А. В. Латышев, А. А. Юшканов, “Точное решение задачи о прохождении тока через границу раздела кристаллитов в металле”, ФTT, 43:10 (2001), 1744-1750.

[3] Х. А. Хачатрян, "О некоторых интегро-дифференциальных уравнениях, возникающих в физической кинетике", Изв. НАН РА. Сер. матем., 39:3 (2004), 72-80.

[4] Х.А. Хачатрян, О факторизачионных методах решения некоторого класса интегральных и интегро-дифференииальных уравнений на полуоси, Дис. ... канд. физ.-матем. наук, Ереван, 2005.

[5] С.Г. Крейн, Ю.Н. Петунин, Е. М. Семенов, Интерполяиия линейных операторов, Наука, М., 1978.

[6] В. С. Владимиров, В.В. Жаринов, Уравнения математической физики, Физматлит, M., 2000.

[7] А.Н. Колмогоров, В. С. Фомин, Элементы теории функиий и функиионального анализа, Наука, М., 1981.

\section{Х. А. Хачатрян}

Поступило

Институт математики НАН Республики Армения, Ереван 22.01.2007

E-mail: aghavard@hotbox.ru 